\title{
Deep brain stimulation in the caudal zona incerta in patients with essential tremor: effects on cognition 1 year after surgery
}

\author{
Johanna Philipson, MS, ${ }^{1}$ Patric Blomstedt, MD, PhD, ${ }^{1}$ Marwan Hariz, MD, PhD, ${ }^{1,2}$ and \\ Marjan Jahanshahi, $\mathrm{PhD}^{2,3}$
}

\begin{abstract}
'Department of Pharmacology and Clinical Neuroscience, Umeå University, Umeå, Sweden; 2Unit of Functional Neurosurgery, UCL Institute of Neurology, London, United Kingdom; and ${ }^{3}$ The Clinical Hospital of Chengdu Brain Science Institute, MOE Key Lab for Neuroinformation, University of Electronic Science and Technology of China, Chengdu, China
\end{abstract}

\begin{abstract}
OBJECTIVE The ventral intermediate nucleus (VIM) of the thalamus is currently the established target in the use of deep brain stimulation (DBS) to treat essential tremor (ET). In recent years, the caudal zona incerta (cZi), a brain target commonly used during the lesional era, has been revived as the primary target in a number of DBS studies that show evidence of the efficacy of cZi targeting in DBS treatment for controlling the symptoms of ET. The authors sought to obtain comprehensive neuropsychological data and thoroughly investigate the cognitive effects of cZi targeting in patients with ET treated with DBS.
\end{abstract}

METHODS Twenty-six consecutive patients with ET who received DBS with cZi as the target at our department from December 2012 to February 2017 were included in this study. All patients were assessed using a comprehensive neuropsychological test battery covering the major cognitive domains both preoperatively and 12 months postoperatively.

RESULTS The results show no major adverse effects on patient performance on the tests of cognitive function other than a slight decline of semantic verbal fluency.

CONCLUSIONS This study indicates that the $c Z i$ is a safe target from a cognitive perspective in the treatment of ET with DBS.

https://thejns.org/doi/abs/10.3171/2019.9.JNS191646

KEYWORDS zona incerta; essential tremor; deep brain stimulation; cognition; functional neurosurgery

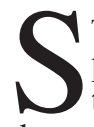
TEREOTACTIC functional neurosurgery with lesional procedures was introduced in $1947 . .^{41}$ Although attempts at stimulation of the brain occurred earlier, 7,21 the marking of a new starting point for deep brain stimulation (DBS) came with a report published in 1987,6 and since then DBS has revolutionized the treatment of movement disorders, in particular Parkinson's disease and essential tremor (ET). ${ }^{11}$

In DBS, different brain structures are targeted depending on the symptoms of the patient. The ventral intermediate nucleus (VIM) of the thalamus is the established target for ET treatment..$^{5,28,30,43}$ In recent years, several studies have, with promising results, revived the caudal zona incerta (cZi), a brain target often used during the lesional era, for use with DBS treatment of ET..$^{1,15,24,34,42}$ This target is situated below the VIM, between the red nucleus and the posteromedial border of the subthalamic nucleus (STN).$^{8,13}$ There is increasing evidence of the efficacy of DBS with cZi as the primary target for controlling the symptoms of ET. $7,18,36$

Cognitive deficits in patients with ET have received increasing attention during the last 2 decades. ${ }^{26}$ There are now several reported studies showing executive difficulties and attention deficits in patients with ET. ${ }^{19,23,32,45}$ The severity of cognitive dysfunction is not necessarily correlated with ET disease severity. However, the same authors demonstrated an association between cortical volume loss and cognitive dysfunction in ET. Bilateral structural

ABBREVIATIONS BVMT-R = Brief Visuospatial Memory Test-Revised; CWIT = Color Word Interference Test; cZi = caudal zona incerta; DBS = deep brain stimulation; ET = essential tremor; ETRS = Essential Tremor Rating Scale; $\mathrm{MCI}=$ mild cognitive impairment; NART-SWE = Swedish version of the National Adult Reading Test; PASAT = Paced Auditory Serial Addition Test; STN = subthalamic nucleus; TMT = Trail Making Test; VIM = ventral intermediate nucleus; VL = ventrolateral nucleus; WAIS-IV = Wechsler Adult Intelligence Scale-IV.

SUBMITTED June 13, 2019. ACCEPTED September 23, 2019.

INCLUDE WHEN CITING Published online December 20, 2019; DOI: 10.3171/2019.9.JNS191646. 
changes were noted in the cerebellum as well as in various cerebral regions. Gray matter volume loss was found in the medial frontal gyrus, anterior cingulate cortex, insula, and postcentral gyrus, all of which are important in highlevel cognitive functions. ${ }^{4}$

There is also increasing interest regarding social cognition and theory of mind in relation to ET. In one study, ${ }^{40}$ cognitive and affective theory of mind in ET patients were investigated. The results showed deficits in cognitive aspects of social cognition, i.e., when patients were asked to deduce and attribute mental states to others. The authors interpreted the results as a consequence of a neurodegenerative process involving a dysfunctional dorsolateral prefrontal cortex-cerebellum circuit. In contrast, the authors of another study found that facial emotion recognition was inversely correlated with tremor severity in ET patients and concluded that their observations needed further investigation to determine whether the deficits were caused by cortical dysfunction or were secondary to abnormal processing in cerebello-cortical loops. ${ }^{2}$

The cognitive effects of DBS treatment in ET have been investigated using a variety of neuropsychological tests in a number of studies (see Table 1 for a summary). For thalamic (VIM) DBS treatment in patients with ET, some studies have shown a slight cognitive improvement in certain cognitive domains and also postoperative cognitive decline in verbal fluency. ${ }^{14,16,33,44}$ In one reported case the authors also found declines in verbal fluency and verbal recall in a patient treated with bilateral VIM stimulation. However, these declines were only found with stimulation turned off. ${ }^{33}$ Some reported studies have found no cognitive change related to DBS in the thalamic area, ${ }^{22,27}$ while others have shown that low-frequency stimulation of the ventrolateral nucleus (VL) has less impact on phonemic and semantic fluency than high-frequency stimulation. ${ }^{35}$ In all of the studies we have reviewed, the authors concluded that thalamic stimulation for ET is safe and does not affect higher cognitive processes.

To our knowledge, the cognitive effects of $\mathrm{cZi}$ as the target for DBS in ET have not previously been thoroughly reported. There has been a previous report of transient decrease of verbal fluency in patients with ET after cZi DBS,${ }^{18}$ but no other cognitive measurements were included in that study. Here, we report comprehensive neuropsychological data on patients with ET with DBS in the cZi.

\section{Methods \\ Study Design}

All 26 patients (14 male, 25 right-handed) with ET who received DBS with $\mathrm{cZi}$ as target at our department from December 2012 to February 2017 were included in this study. Their mean $( \pm$ SD) age was $65.8 \pm 6.66$ years, with $12 \pm 2.95$ years of education. The average duration of ET was $30.6 \pm 20.1$ years. Patients with other preexisting conditions were excluded as well as patients who had already had DBS surgery in other targets and had additional electrodes implanted in the $\mathrm{cZi}$ during the inclusion period. Both unilateral $(\mathrm{n}=18$ left $\mathrm{cZi}, 2$ right $\mathrm{cZi})$ and bilateral $(n=6)$ procedures were included. In total, 26 patients consecutively treated with DBS at our center were included.
All patients were assessed at baseline and 12 months postoperatively according to the Essential Tremor Rating Scale (ETRS). This rating scale is a compound rating scale with separate sections for impairment, disability, and activities of daily living (ADL). ${ }^{17}$

In our sample, 3 patients fulfilled criteria for mild cognitive impairment $(\mathrm{MCI})$. One patient received bilateral DBS and the other 2 patients received a unilateral DBS treatment procedure. We chose to use the MCI consensus criteria established by the Movement Disorder Society Task Force Guidelines. ${ }^{31}$ MCI was therefore considered to be present when impairments (test results a minimum of 1 SD below normal performance of nonimpaired individuals) were found on at least 2 tests within a single cognitive domain, with other domains unimpaired, or on at least 1 test in 2 or more cognitive domains.

\section{Surgical Procedure}

Stereotactic implantation of the DBS electrodes 3389 (Medtronic) was performed using the Leksell frame model G (Elekta Instruments). To identify and target the cZi (slightly posteromedial to the tail of the STN, at the axial level of the maximal diameter of the red nuclei), stereotactic T2-weighted 1.5-T MRI sequences were used (Fig. 1). Typically, the center of the deepest contact was targeted $4 \mathrm{~mm}$ below the anterior commissure-posterior commissure line and $12 \mathrm{~mm}$ lateral and $7.5 \mathrm{~mm}$ posterior to the midcommissural point. The same surgeon (P.B.) performed all surgical procedures.

\section{Neuropsychological Evaluation}

All patients were assessed by the same senior neuropsychologist (J.P.) preoperatively and 12 months postoperatively. The major cognitive domains were assessed by using the following tests.

\section{Global Cognitive Function}

Ravens colored matrices is a nonverbal measure of intelligence and global mental ability and nonverbal reasoning with a maximum score of 36 . Results are presented in percentiles standardized based on age group. ${ }^{37}$

A Swedish version of the National Adult Reading Test (NART-SWE) is designed to estimate premorbid IQ with the use of irregularly spelled and pronounced words. The patient is asked to read the words aloud and from the number of correct pronunciations an estimate of premorbidity was obtained. The results are presented as index scores, with a mean score of $100 \pm 15 .^{38}$

\section{Memory}

The Claeson-Dahls test is a test of verbal learning and memory (retention) administered in Swedish. Ten words are read out loud to the patient, and after a 15-second pause, the patient recalls as many as possible. This portion of the test is completed after a maximum of 10 repetitions, or when the patient has succeeded in recalling all 10 words correctly 2 consecutive times. After 30 minutes the patient is asked to again recall as many words as possible. In addition to delayed recall, a delayed recognition task is also administered. Four alternate versions are available for 


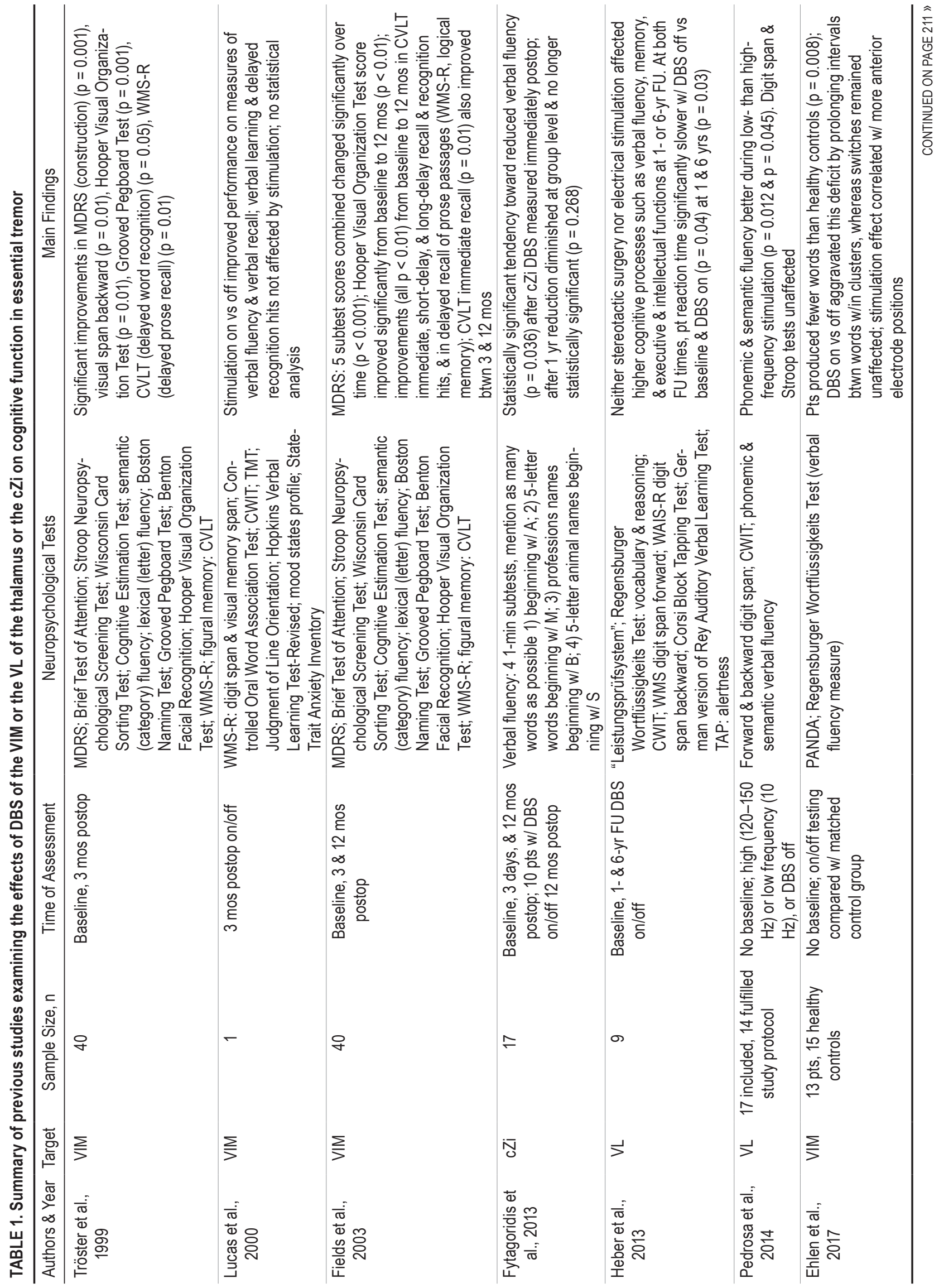




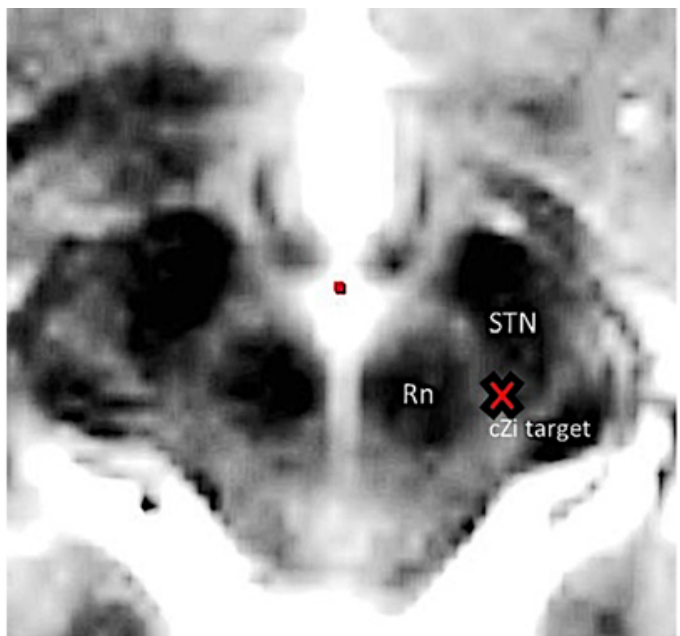

FIG. 1. The target point in the cZi (red X), medial to the tail of the STN. $\mathrm{Rn}=$ red nucleus. Figure is available in color online only.

retest conditions. Results are calculated based on the number of words recalled and the number of times the list of words needs to be repeated. A low score indicates a faster learning curve. ${ }^{10}$

The Brief Visuospatial Memory Test-Revised (BVMT$\mathrm{R}$ ) is a test for assessment of visual memory and comprises 6 alternate, equivalent forms (forms 1 through 6). Each form consists of 6 geometrical figures and 12 recognition items. The figures are presented for a 10 -second period, after which the participant is asked to draw as many geometrical figures as possible, preferably matching the stimulus card with regard to placement and accuracy. The figures are presented 3 times to assess for short-term spatial memory and learning. After 30 minutes, delayed recall is tested, followed by a delayed recognition task during which the patient is presented with 12 figures, of which 6 are the original figures presented previously. Scores are given for accuracy and placement of the figures. Scores range from 0 to 12 in each of the 3 trials, and thus the maximum scores are 36 in the learning task and 12 in the recognition task. Higher scores indicate better visual memory. ${ }^{3}$

The Wechsler Adult Intelligence Scale-IV (WAIS-IV) digit span forward and digit span backward are tests of short-term verbal memory and verbal working memory. Digit sequences are presented to the patient starting with a length of 2 digits. Testing stops when the patient incorrectly reports both trials at 1 sequence length, or when all sequences are reported correctly ( 9 digits forward, 8 backward). Scores range from 0 to 18 forward and 0 to 16 backward, with higher scores indicating better performance. Raw scores are converted to age-corrected scaled scores, ranging from 1 to 19 , with higher scores indicating better performance. ${ }^{47}$

\section{Executive Function}

The Delis-Kaplan Executive Function System (DKEFS) is a set of 9 standardized subtests for assessing higher-level cognitive functions, referred to as executive functions. ${ }^{12}$ 
The Trail Making Test (TMT), a D-KEFS subtest, is used to assess visual attention, behavioral regulation, task switching, and processing speed. The test consists of 5 individual subtests: 1) visual scanning, in which the participant marks all circles with the number " 3 " presented among both letters and other numbers; 2) number sequencing, in which the participant connects all numbers in successive order, ignoring any letters on the sheet; 3 ) letter sequencing, in which the participant connects letters in alphabetical order, ignoring any numbers on the sheet; 4) number-letter switching, in which the participant connects circles by switching between digits and letters (1-A-2-B-... etc.) in an alternating sequence; and 5) motor speed, in which as a measure of general motor speed the participant traces a dashed line connecting empty circles as fast as possible. Executive functions believed to be important for successful completion of this test are behavioral regulation, cognitive flexibility, and inhibition of perseverative responding. Scores are based on the time needed to complete the individual subtests. Results are transformed to scaled scores, ranging from 1 to 19 , and a higher score indicates better performance.

\section{Verbal Fluency}

The verbal functioning test consisted of the following 3 tasks: phonemic fluency, semantic fluency, and switching category fluency. For each subtest, participants are given 1 minute to produce as many unique words as possible within a semantic category or starting with a given letter. The participant's score in each task is the number of unique correct words, excluding intrusion and repetition errors. In the switching category task the participant is asked to shift between semantic categories. Two alternative versions of the test are available. Raw scores are transformed into scaled scores ranging from 1 to 19 , and a higher score indicates better performance.

The Stroop Color Word Interference Test (CWIT) includes the following 4 subtests: 1) color naming: naming the colors of colored rectangles; 2) word reading: reading the color words "red," "green," or "blue" printed in black ink; 3) color/word interference/inhibition: naming the color of color words printed in incongruent ink, for example, the word "red" printed in blue ink. These subtests assess the participant's ability to inhibit the more habitual response of reading the words to engage in the alternative response of naming the color of ink in which they are printed. 4) Inhibition switching: the same conditions as subtest 3 , except when there is a box around the word, the participant has to read the word. Thus, subtest 4 requires both the inhibition of habitual responses and a switching response strategy across trials. The total time to complete each of the subtests and the number of self-corrected and uncorrected errors are recorded. Scores are transformed into scaled scores ranging from 1 to 19. A higher score indicates a better result.

\section{Attention}

The dichotic listening task is a test for studying brain asymmetry in auditory processing. Different sounds are sent to the participant's right and left ear at the same time. Three conditions are used to assess different kinds of cog- nitive processes: 1) a nonforced condition normally used to determine speech lateralization, 2) a forced-right condition focusing on attention processes, and 3) a forced-left condition evaluating an executive cognitive control process. The results from the 2 forced-attention conditions are calculated as correct score percentages of the 30 dichotic stimuli presented separately to the right and left ear. The raw score is transformed to a t score ranging from $<20$ to $>80$. Interpretations of the $t$ scores are made with respect to the condition being tested and indicate, for instance, the patient's ability to shift auditory attention. ${ }^{25}$

The Paced Auditory Serial Addition Test (PASAT) assesses attention, working memory, and speed of auditory information processing, as well as calculation ability. To ensure standardization in the rate of stimulus presentation, the test is administered using a prerecorded audio tape. Every 3 seconds, single digits are presented and the patient is asked to add each new digit to the one presented directly prior to it and verbally report the sum. The correct answers given constitute the raw score, which is then transformed to percentiles and z scores. Higher scores indicate better performance.

\section{Statistical Analysis}

Statistical analyses of neuropsychological data were performed on normative scores corrected for age, sex, and/or education. These results are presented in Table 2 . Since the data were not normally distributed, the Wilcoxon signed-rank test for paired samples was used to compare pre- and postoperative scores. A p value $<0.05$ was considered statistically significant.

\section{Results}

The total ETRS score at baseline was $46.32 \pm 14.08$. At 12 months follow-up, the total ETRS score on stimulation was $17.0 \pm 9.48(\mathrm{p}=0.0001)$ and off stimulation was 53.26 $\pm 15.57(\mathrm{p}=0.11)$, indicating significant improvement of ET after surgery with stimulation on.

Measures of global cognition were only used preoperatively to ensure eligibility for the procedure. During the course of enrollment, we changed from using Ravens matrices to the NART-SWE due to the lack of updated normative data. The results of both tests show mean results within the normal range (Ravens matrices $56.75 \pm 29.93$, NART-SWE $105.43 \pm 9.99$ ).

The pre- and postoperative patient results on the cognitive measures are shown in Table 2 . The only cognitive test that showed a significant decline at the 12-month follow-up relative to the preoperative assessment was semantic verbal fluency $(\mathrm{p}=0.027)$. None of the other measures of cognitive function showed any significant change from pre- to postoperative assessment. We found no differences in change in the cognitive measures from pre- to postoperative assessment when we compared the 6 patients with bilateral DBS with the 19 patients with unilateral DBS.

\section{Discussion}

ETRS scores show a marked improvement 12 months after cZi DBS, with a symptom reduction of almost $63 \%$ 
TABLE 2. Scores on tests of cognitive function before (at baseline) and 12 months after surgery

\begin{tabular}{|c|c|c|c|}
\hline Test & Baseline Score & 12-Mo Score & $p$ Value at Baseline vs 12 Mos \\
\hline \multicolumn{4}{|l|}{ Memory } \\
\hline \multicolumn{4}{|l|}{ Claeson-Dahls test (t score) } \\
\hline Verbal learning & $46.60 \pm 9.99$ & $44.72 \pm 11.46$ & 0.193 \\
\hline Retention & $43.52 \pm 11.90$ & $47.52 \pm 12.60$ & 0.117 \\
\hline \multicolumn{4}{|l|}{ BVMT-R (t score) } \\
\hline Recall & $49.68 \pm 13.70$ & $48.92 \pm 9.22$ & 0.987 \\
\hline Learning & $49.36 \pm 8.08$ & $51.69 \pm 9.16$ & 0.344 \\
\hline Delayed recall & $48.56 \pm 15.11$ & $49.92 \pm 10.33$ & 0.566 \\
\hline WAIS-IV digit span forward (scaled score) & $9.69 \pm 2.80$ & $10.04 \pm 3.13$ & 0.710 \\
\hline \multicolumn{4}{|l|}{ Executive function } \\
\hline TMT, shifting (scaled score) & $10.52 \pm 2.24$ & $10.85 \pm 2.66$ & 0.135 \\
\hline TMT, shifting total errors (scaled score) & $10.68 \pm 1.25$ & $10.38 \pm 2.12$ & 0.851 \\
\hline \multicolumn{4}{|l|}{ Verbal fluency } \\
\hline Phonemic fluency (scaled score) & $10.08 \pm 3.63$ & $9.27 \pm 4.20$ & 0.061 \\
\hline Semantic fluency (scaled score) & $11.77 \pm 4.09$ & $10.46 \pm 4.18$ & $0.027^{*}$ \\
\hline Switching category fluency (scaled score) & $10.27 \pm 3.00$ & $9.76 \pm 3.09$ & 0.210 \\
\hline Correct switches (scaled score) & $10.62 \pm 2.45$ & $10.24 \pm 2.65$ & 0.385 \\
\hline \multicolumn{4}{|l|}{ CWIT (scaled score) } \\
\hline Inhibition & $9.73 \pm 3.31$ & $9.96 \pm 3.01$ & 0.675 \\
\hline Shifting & $9.23 \pm 3.33$ & $9.64 \pm 3.34$ & 0.399 \\
\hline Inhibition errors & $10.73 \pm 2.39$ & $10.88 \pm 2.25$ & 0.425 \\
\hline Shifting errors & $9.50 \pm 3.85$ & $10.68 \pm 3.17$ & 0.059 \\
\hline \multicolumn{4}{|l|}{ Attention/working memory } \\
\hline PASAT (z score) & $-0.53 \pm 0.78$ & $-0.46 \pm 0.93$ & 0.553 \\
\hline \multicolumn{4}{|l|}{ Dichotic listening (t score) } \\
\hline Nonforced RE & $53.57 \pm 10.61$ & $50.75 \pm 10.74$ & 0.247 \\
\hline Nonforced LE & $43.50 \pm 6.71$ & $42.75 \pm 10.57$ & 0.442 \\
\hline Forced right $\mathrm{RE}$ & $51.64 \pm 9.63$ & $48.55 \pm 11.01$ & 0.152 \\
\hline Forced right LE & $48.79 \pm 7.51$ & $46.18 \pm 10.02$ & 0.953 \\
\hline Forced left RE & $58.07 \pm 9.78$ & $58.33 \pm 7.99$ & 0.919 \\
\hline Forced left LE & $43.93 \pm 6.96$ & $42.08 \pm 7.56$ & 0.959 \\
\hline WAIS-IV digit span backward (scaled score) & $9.46 \pm 2.85$ & $9.65 \pm 3.60$ & 0.569 \\
\hline
\end{tabular}

$\mathrm{LE}=$ left ear; $\mathrm{RE}=$ right ear.

Values are presented as mean \pm SD unless otherwise indicated.

${ }^{*}$ Statistically significant $(p<0.05)$.

compared to symptoms present at the presurgical baseline, results indicating an effective alleviation of motor symptoms. In this sample, we found no significant changes of main cognitive domains assessed (memory, executive function, attention) 12 months after cZi DBS compared to the presurgical baseline. When the group with MCI at baseline $(n=3)$ was evaluated postoperatively, the results were similar to those found in the non-MCI group.

The only significant adverse finding in the study patients was a decrease in semantic fluency postoperatively. Decline in verbal fluency has previously been reported following STN DBS and globus pallidus internus DBS in patients with Parkinson's disease, which has been described in a rather comprehensive meta review. ${ }^{46}$ Decline in verbal fluency has also been reported after VIM DBS in ET, as mentioned previously..$^{14,16,33,44}$ So far there is no consensus as to what causes this deterioration. In line with the results from these earlier studies, we too noted a slight decrease in phonemic fluency, but this did not reach significance in our sample. Since we did not conduct repeated testing with stimulation on versus off, we cannot determine whether the decline in verbal fluency was caused by the surgical procedure or by the DBS. A previous study ${ }^{18}$ found that the detected effects on verbal fluency did not increase with stimulation of the cZi. Thus, rather than being a result of stimulation of the cZi itself, the changes observed on verbal fluency could have been the consequence of a secondary influence on other structures. Any eventual effect of the surgery (i.e., insertion of DBS lead, edema) should dissipate within 2-3 weeks after the surgery and would not 
be present at the 12-month follow-up. All surgeries in the present study involved only 1 pass of the DBS electrode and were conducted without the use of microelectrode recording. However, this study cannot settle the issue of whether the surgical procedure or the stimulation caused the decline in verbal fluency in our sample.

Even though similar side effects have been reported in both VIM and STN DBS, we posit that cZi should be considered an important and perhaps, at times, superior target in the treatment of tremor in ET. Several VIM DBS studies have been found to have the active contacts situated below the VIM in the zona incerta, including the cZi. STN stimulation also seems to be most effective in the area between the upper STN (sensorimotor part) and the subthalamic area. Structures of importance in this area are STN projections, fields of Forel, and the zona incerta. It has also been pointed out that DBS in the cZi is more efficient than in the VIM, since the same amount of tremor reduction can be attained with lower energy consumption, hence prolonging battery life., 920,39

Our sample is relatively small. This may of course affect our results, especially when comparing the bilateral and unilateral cases, as well as the impact of MCI at baseline. We must therefore be careful when drawing conclusions from these small groups.

The experience we have obtained during the years of providing cZi DBS has had a great impact on the neuropsychological methods we now use at our unit. Since continuous performance tests measuring attention involve a motor component, such as the integrated visual and auditory (IVA) continuous performance tests (CPTs) CPT-3 or IVA++, we decided not to include these kinds of tests for the assessment of attention. However, the use of the PASAT has its own set of challenges, and it is well known that performance on this test is easily affected by stress. ${ }^{29}$ The use of auditory-based tests for attention, such as the dichotic listening test, might at first seem to solve this problem, but the problem of hearing loss in an elderly population gives rise to a new set of challenges when interpreting the results. This can make the use of similar auditory-based tests practically impossible since the patients cannot distinguish various sounds due to a slight loss of hearing, which at times may not even be detected by the patient.

\section{Conclusions}

This study indicates that the cZi is a safe DBS target from a cognitive perspective. We did not find any major negative effects on the tests of cognitive function included, other than a slight decline of semantic verbal fluency.

\section{References}

1. Andy OJ, Jurko MF, Sias FR Jr: Subthalamotomy in treatment of Parkinsonian tremor. J Neurosurg 20:860-870, 1963

2. Auzou N, Foubert-Samier A, Dupouy S, Meissner WG: Facial emotion recognition is inversely correlated with tremor severity in essential tremor. J Neural Transm (Vienna) 121:347-351, 2014

3. Benedict RHB: Brief Visuospatial Memory Test-Revised. Odessa, FL: Psychological Assessment Resources, Inc., 1997

4. Bhalsing KS, Upadhyay N, Kumar KJ, Saini J, Yadav R, Gupta AK, et al: Association between cortical volume loss and cognitive impairments in essential tremor. Eur J Neurol 21:874-883, 2014

5. Benabid AL, Pollak P, Gervason C, Hoffmann D, Gao DM, Hommel M, et al: Long-term suppression of tremor by chronic stimulation of the ventral intermediate thalamic nucleus. Lancet 337:403-406, 1991

6. Benabid AL, Pollak P, Louveau A, Henry S, de Rougemont $\mathrm{J}$ : Combined (thalamotomy and stimulation) stereotactic surgery of the VIM thalamic nucleus for bilateral Parkinson disease. Appl Neurophysiol 50:344-346, 1987

7. Blomstedt P, Hariz MI: Deep brain stimulation for movement disorders before DBS for movement disorders. Parkinsonism Relat Disord 16:429-433, 2010

8. Blomstedt P, Sandvik U, Fytagoridis A, Tisch S: The posterior subthalamic area in the treatment of movement disorders: past, present, and future. Neurosurgery 64:1029-1042, 2009

9. Blomstedt P, Sandvik U, Linder J, Fredricks A, Forsgren L, Hariz MI: Deep brain stimulation of the subthalamic nucleus versus the zona incerta in the treatment of essential tremor. Acta Neurochir (Wien) 153:2329-2335, 2011

10. Claeson LE, Esbjörnsson E, Tännérus BM, Wahlbin M: Claeson-Dahls test för inlärning och minne. Reviderad version manual. Stockholm: Psykologiförlaget, 1998

11. Clausen J: Ethical brain stimulation - neuroethics of deep brain stimulation in research and clinical practice. Eur J Neurosci 32:1152-1162, 2010

12. Delis DC, Kaplan E, Kramer JH: D-KEFS. Svenskt manualsupplement. Stockholm: Psykologiförlaget, 2005

13. Deuschl G, Raethjen J, Hellriegel H, Elble R: Treatment of patients with essential tremor. Lancet Neurol 10:148-161, 2011

14. Ehlen F, Vonberg I, Tiedt HO, Horn A, Fromm O, Kühn AA, et al: Thalamic deep brain stimulation decelerates automatic lexical activation. Brain Cogn 111:34-43, 2017

15. Fager CA: Evaluation of thalamic and subthalamic surgical lesions in the alleviation of Parkinson's disease. J Neurosurg 28:145-149, 1968

16. Fields JA, Tröster AI, Woods SP, Higginson CI, Wilkinson SB, Lyons KE, et al: Neuropsychological and quality of life outcomes 12 months after unilateral thalamic stimulation for essential tremor. J Neurol Neurosurg Psychiatry 74:305311, 2003

17. Fahn S, Tolosa E, Marin C: Clinical rating scale for tremor, in Jankovic J, Tolosa E (eds): Parkinson's Disease and Movement Disorders. Munich: Urban \& Schwarzenberg, 1993, pp 271-280

18. Fytagoridis A, Sjöberg RL, Åström M, Fredricks A, Nyberg L, Blomstedt P: Effects of deep brain stimulation in the caudal zona incerta on verbal fluency. Stereotact Funct Neurosurg 91:24-29, 2013

19. Gasparini M, Bonifati V, Fabrizio E, Fabbrini G, Brusa L, Lenzi GL, et al: Frontal lobe dysfunction in essential tremor: a preliminary study. J Neurol 248:399-402, 2001

20. Hamel W, Fietzek U, Morsnowski A, Schrader B, Herzog J, Weinert D, et al: Deep brain stimulation of the subthalamic nucleus in Parkinson's disease: evaluation of active electrode contacts. J Neurol Neurosurg Psychiatry 74:1036-1046, 2003

21. Hariz MI, Blomstedt P, Zrinzo L: Deep brain stimulation between 1947 and 1987: the untold story. Neurosurg Focus 29(2):E1, 2010

22. Heber IA, Coenen VA, Reetz K, Schulz JB, Hoellig A, Fimm $B$, et al: Cognitive effects of deep brain stimulation for essential tremor: evaluation at 1 and 6 years. J Neural Transm (Vienna) 120:1569-1577, 2013

23. Higginson CI, Wheelock VL, Levine D, King DS, Pappas CTE, Sigvardt KA: Cognitive deficits in essential tremor consistent with frontosubcortical dysfunction. J Clin Exp Neuropsychol 30:760-765, 2008 
24. Houdart R, Mamo H, Dondey M, Cophignon J: [Results of subthalamic coagulations in Parkinson's disease (apropos of 50 cases).] Rev Neurol (Paris) 112:521-529, 1965 (French)

25. Hugdahl K, Asbjørnsen A: Dikotisk lyssning med CV-stavelser: Manual. Stockholm: Psykologiförlaget, 1994

26. Janicki SC, Cosentino S, Louis ED: The cognitive side of essential tremor: what are the therapeutic implications? Ther Adv Neurol Disorder 6:353-368, 2013

27. Klein J, Büntjen L, Jacobi G, Galazky I, Panther P, Zaehle $\mathrm{T}$, et al: Bilateral thalamic deep brain stimulation for essential tremor in elderly patients. J Neural Transm (Vienna) 124:1093-1096, 2017

28. Koller W, Pahwa R, Busenbark K, Hubble J, Wilkinson S, Lang A, et al: High-frequency unilateral thalamic stimulation in the treatment of essential and parkinsonian tremor. Ann Neurol 42:292-299, 1997

29. Lezak MD, Howieson DB, Bigler ED, Tranel D: Neuropsychological Assessment, ed 5. New York: Oxford University Press, 2012

30. Limousin P, Speelman JD, Gielen F, Janssens M: Multicentre European study of thalamic stimulation in parkinsonian and essential tremor. J Neurol Neurosurg Psychiatry 66:289296, 1999

31. Litvan I, Goldman JG, Tröster AI, Schmand BA, Weintraub D, Petersen RC, et al: Diagnostic criteria for mild cognitive impairment in Parkinson's disease: Movement Disorder Society Task Force guidelines. Mov Disord 27:349-356 2012

32. Lombardi WJ, Woolston DJ, Roberts JW, Gross RE: Cognitive deficits in patients with essential tremor. Neurology 57:785-790, 2001

33. Lucas JA, Rippeth JD, Uitti RJ, Shuster EA, Wharen RE: Neuropsychological functioning in a patient with essential tremor with and without bilateral VIM stimulation. Brain Cogn 42:253-267, 2000

34. Mundinger F: [Subthalamotomy for the treatment of extrapyramidal movement disorders.] Dtsch Med Wochenschr 90:2002-2007, 1965 (German)

35. Pedrosa DJ, Auth M, Pauls KAM, Runge M, Maarouf M, Fink GR, et al: Verbal fluency in essential tremor patients: the effects of deep brain stimulation. Brain Stimul 7:359_ 364, 2014

36. Plaha P, Javed S, Agombar D, O' Farrell G, Khan S, Whone A, et al: Bilateral caudal zona incerta nucleus stimulation for essential tremor: outcome and quality of life. J Neurol Neurosurg Psychiatry 82:899-904, 2011

37. Raven J, Raven JC, Court JH: Manual for Raven's progressive matrices and vocabulary scales. Section 2: The coloured progressive matrices. San Antonio: Harcourt Assessment Inc., 1998

38. Rolstad S, Nordlund A, Gustavsson MH, Eckerström C, Klang O, Hansen S, et al: The Swedish National Adult Reading Test (NART-SWE): a test of premorbid IQ. Scand J Psychol 49:577-582, 2008
39. Sandvik U, Koskinen LO, Lundquist A, Blomstedt P: Thalamic and subthalamic deep brain stimulation for essential tremor: where is the optimal target? Neurosurgery 70:840846,2012

40. Santangelo G, Trojano L, Barone P, Errico D, Improta I, Agosti V, et al: Cognitive and affective theory of mind in patients with essential tremor. J Neurol 260:513-520, 2013

41. Spiegel EA, Wycis HT, Marks M, Lee AJ: Stereotaxic apparatus for operations on the human brain. Science 106:349350, 1947

42. Story JL, French LA, Chou SN, Meier MJ: Experiences with subthalamic lesions in patients with movement disorders. Confin Neurol 26:218-221, 1965

43. Sydow O, Thobois S, Alesch F, Speelman JD: Multicentre European study of thalamic stimulation in essential tremor: a six year follow up. J Neurol Neurosurg Psychiatry 74:1387-1391, 2003

44. Tröster AI, Fields JA, Pahwa R, Wilkinson SB, Strait-Tröster KA, Lyons K, et al: Neuropsychological and quality of life outcome after thalamic stimulation for essential tremor. Neurology 53:1774-1780, 1999

45. Tröster AI, Woods SP, Fields JA, Lyons KE, Pahwa R, Higginson CI, et al: Neuropsychological deficits in essential tremor: an expression of cerebello-thalamo-cortical pathophysiology? Eur J Neurol 9:143-151, 2002

46. Wang JW, Zhang YQ, Zhang XH, Wang YP, Li JP, Li YJ: Cognitive and psychiatric effects of STN versus GPi deep brain stimulation in Parkinson's disease: a meta-analysis of randomized controlled trials. PLoS One 11:e0156721, 2016

47. Wechsler D: Wechsler Adult Intelligence Scale-Fourth Edition. Swedish Version. Stockholm: Pearson Inc., 2010

\section{Disclosures}

Dr. Blomstedt is a consultant for Abbott, Boston Scientific, and Medtronic and is a stockholder in Mithridaticum AB. Dr. Hariz has received honoraria and travel expenses from Medtronic and Boston Scientific for speaking at meetings.

\section{Author Contributions}

Conception and design: Philipson, Jahanshahi. Acquisition of data: Philipson. Analysis and interpretation of data: Philipson. Drafting the article: Philipson, Jahanshahi. Critically revising the article: all authors. Reviewed submitted version of manuscript: all authors. Approved the final version of the manuscript on behalf of all authors: Philipson. Statistical analysis: Philipson. Study supervision: Blomstedt, Hariz, Jahanshahi. Primary surgeon: Blomstedt.

\section{Correspondence}

Johanna Philipson: University Hospital of Umeå, Sweden. johanna.philipsson@umu.se. 